\title{
Corneal Edema with Anterior Uveitis after Exposure to the Sap of Euphorbia trigona: A Case Report
}

\author{
Takashi Ono ${ }^{a, b}$ Yosai Moria Ryohei Nejima ${ }^{a}$ Takuya Iwasaki ${ }^{a}$ \\ Takashi Miyai $^{b}$ Shinichiro Ohtani ${ }^{a, c}$ Kazunori Miyata ${ }^{a, c}$ \\ aDepartment of Ophthalmology, Miyata Eye Hospital, Miyazaki, Japan; bepartment of \\ Ophthalmology, Graduate School of Medicine, The University of Tokyo, Tokyo, Japan; \\ 'Department of Ophthalmology, Kagoshima Miyata Eye Clinic, Kagoshima, Japan
}

\section{Keywords}

Euphorbia trigona $\cdot$ Case report $\cdot$ Plant toxin $\cdot$ Keratitis $\cdot$ Anterior uveitis

\begin{abstract}
Although a few cases of dermatitis or keratitis caused by exposure to the sap of Euphorbia trigona have been reported, we present a rare case of transient corneal endothelial dysfunction following exposure to the sap, resulting in corneal edema. A woman in her 70s complained of reduced vision, redness, and teariness in her left eye 2 days after exposure to the sap of E. trigona at home. Upon examination, hyperemia, serious corneal edema, and anterior uveitis with hypopyon were observed in her left eye, without corneal epithelial defects or keratic precipitates. The best-corrected visual acuity (BCVA) was 2.0 (logarithm of the minimum angle of resolution), and the measured central corneal thickness (CCT) was $812 \mu \mathrm{m}$. The patient was treated with topical instillation of $1.5 \%$ levofloxacin and $0.1 \%$ dexamethasone to reduce intraocular inflammation and corneal edema. Three weeks later, the BCVA reached 0 , the CCT was $519 \mu \mathrm{m}$, and the corneal endothelial cell density was 3,233 cells $/ \mathrm{mm}^{2}$. Six months after the injury, the patient had good visual acuity, and the cornea was completely transparent. No recurrence of corneal edema or anterior uveitis was observed. Exposure to the sap of E. trigona can lead to severe corneal edema with anterior uveitis, impairing visual acuity. Taking precautions to prevent the exposure of the eye to the sap of this plant is crucial.
\end{abstract}




\section{Introduction}

Euphorbia trigona, widely known as the African milk tree, is a succulent garden plant grown worldwide. It contains a unique wide stem with numerous thorns. Owing to its size and resistance to pests, it is popular among people with gardening as a hobby. However, it contains a milky sap, which may irritate the skin resulting in dermatitis [1,2]. The Euphorbia genus includes over 2,000 plants in a variety of sizes and forms, and the toxic effect of the sap depends on the species [3]. The toxin of E. trigona was used as a poison to catch fish in Southeast Asia in ancient times [4]. The toxic effect of the sap of the Euphorbia genus on the eyes has been previously reported [5-10], including a few cases of keratitis caused by exposure to the sap of E. trigona [11,12]. Although an acute case of exposure of the eye to the sap of Euphorbia spp. led to corneal blindness requiring corneal transplantation in an untreated patient [13], none of the previous studies have reported changes in corneal thickness, reflecting corneal endothelial dysfunction due to exposure to the sap of E. trigona. Herein, we describe a case of corneal endothelial dysfunction and anterior uveitis after exposure to the sap of E. trigona.

\section{Case Report/Case Presentation}

A woman in her 70s was referred to our clinic complaining of redness and teariness in her left eye. She recounted that the sap of an E. trigona plant at home (Fig. 1a) had splashed into her left eye when she had cut the plant. Two days after the incident, she experienced pain and visual problems and visited a clinic in her neighborhood, where the physician immediately referred her to our hospital. She did not have a history of allergies and did not have any contact with chemicals. Her medical history included a cataract surgery performed 3 years earlier, and she was using a topical instillation for dry eye. Her last best-corrected visual acuity (BCVA) was 0 in terms of the logarithm of the minimum angle of resolution, and she had no other history of ophthalmological or medical problems.

On general examination, her condition was found to be stable. Slit-lamp examination revealed hyperemia with a thick and clouded cornea in her left eye (Fig. 1b-d). There was hypopyon inside the anterior chamber, but no keratic precipitates were observed (Fig. 1b). Corneal epithelial defects were not detected after fluorescein staining (Fig. 1c). The BCVA was 2.0 as measured under photopic conditions. The intraocular pressure was $17 \mathrm{~mm} \mathrm{Hg}$, as measured using a noncontact tonometer, and the central corneal thickness (CCT) was 812 $\mu \mathrm{m}$, as measured using a contact ultrasonic pachymeter. The fundus of the left eye could not be seen because of the clouded cornea and intraocular inflammation; however, ocular ultrasound examination revealed no retinal detachment or vitreous opacity. There was no sign of general inflammation in the blood test results.

We started treatment with topical 1.5\% levofloxacin and $0.1 \%$ dexamethasone 4 times a day. She responded well to treatment showing a reduction in hypopyon and corneal edema the next day. The measured CCT was $744 \mu \mathrm{m}$. She experienced no pain, her BCVA had improved to 1.0, and intraocular pressure was $14 \mathrm{~mm} \mathrm{Hg}$. A week later, the CCT was $621 \mu \mathrm{m}$, and BCVA increased to 0.22 , despite the presence of intraocular cells (grade $3+$ ). Corneal edema and Descemet membrane folds were still present (Fig. 2a), but no corneal epithelial defect was observed upon fluorescein staining (Fig. 2b). We were not able to assess the corneal endothelial cell density (ECD) by noncontact specular microscopy owing to corneal edema. The blood samples collected upon arrival tested negative for viral infections including cytomegalovirus, varicella-zoster virus, and herpes simplex virus. Therefore, we continued the topical instillation at the same frequency of administration for another week.

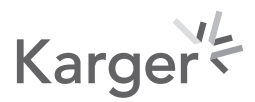




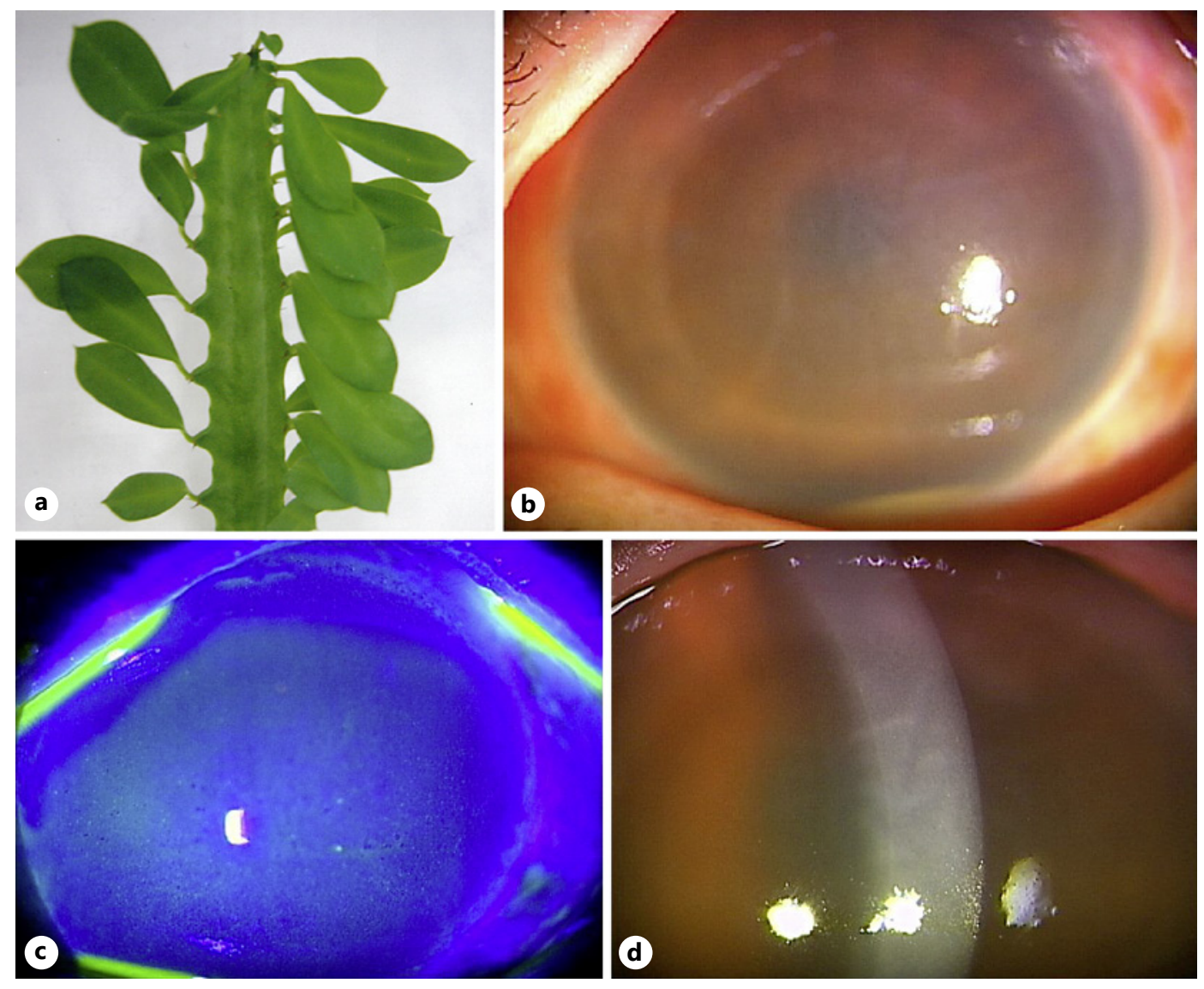

Fig. 1. Corneal edema and anterior uveitis after exposure to the sap of Euphorbia trigona. a A photo of Euphorbia trigona. b Thickened cornea and folds in the Descemet membrane observed on slit-lamp light examination of the anterior chamber at the initial presentation. c No corneal epithelial defect observed after fluorescein staining of the cornea. d Magnified image of the cornea with slit-lamp light examination. Hyperemia, corneal edema, and hypopyon observed in the anterior segment of the eye on the day of the visit.

Two weeks after the injury, the corneal edema recovered with the CCT reaching 547 $\mu \mathrm{m}$. The cornea became clear, and the BCVA recovered to 0.097. Hypopyon disappeared completely with a small number of intraocular cells floating in the anterior chamber (grade $1+$ ). The ECD remained at 3,280 cells $/ \mathrm{mm}^{2}$, the coefficient of variation of the cell area (CV) was $45 \%$, and the proportion of hexagonal cells (6A\%) was $44 \%$. Therefore, the frequency of drug administration was reduced to 3 times a day for another week when the BCVA reached 0 and the CCT was $519 \mu \mathrm{m}$. The frequency of topical instillation of $1.5 \%$ levofloxacin and $0.1 \%$ dexamethasone was subsequently decreased to twice a day for another week. The CCT reached $528 \mu \mathrm{m}$, and the ECD remained at 3,233 cells $/ \mathrm{mm}^{2} 1$ month after the injury; the CV was $46 \%$, and the $6 \mathrm{~A} \%$ was $25 \%$. The anterior segment showed no intraocular inflammation with a completely clear cornea (Fig. 2c, d), and palisades of Vogt were observed around the corneal epithelium (Fig. 2c). Hence, topical instillation of steroids and antibiotics was discontinued. The time-dependent changes in CCT and BCVA are summarized in Figure 3.

A follow-up examination 6 months after the injury revealed a completely transparent cornea with good visual acuity (BCVA; 0); uveitis did not recur during the follow-up period. The ECD remained at 3,244 cells $/ \mathrm{mm}^{2}$, the $\mathrm{CV}$ was $26 \%$, and the $6 \mathrm{~A} \%$ was $66 \%$, indicating no endothelial damage or complications. 


\section{Case Reports in Ophthalmology}
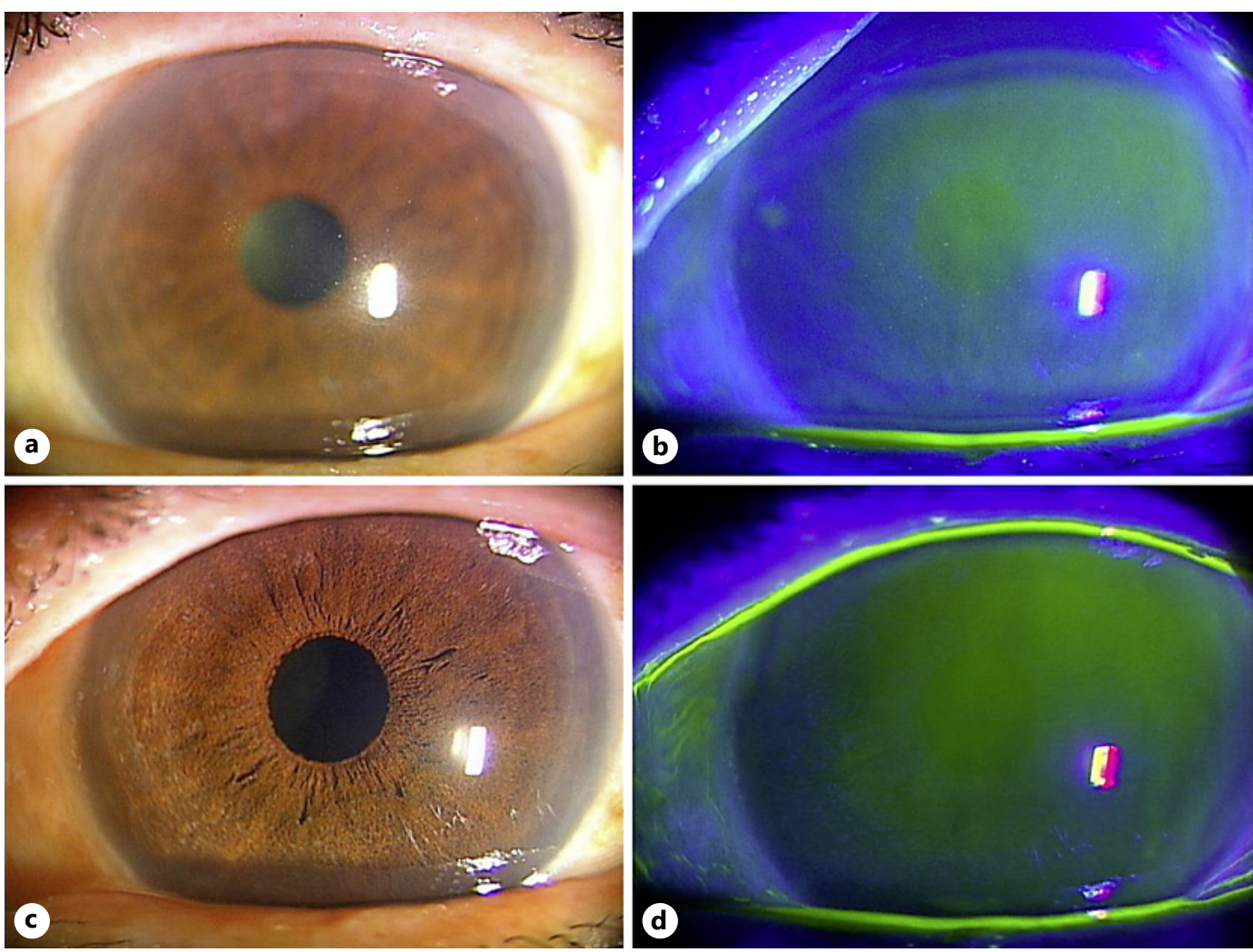

Fig. 2. Changes in the anterior segment of the eye after exposure to the sap of Euphorbia trigona. a The anterior segment of the eye 1 week after the injury. Hyperemia has resolved after treatment, but corneal edema persists. b No corneal epithelial defect observed 1 week after injury following fluorescein staining. c Examination of the anterior segment of the eye revealing complete resolution of corneal edema without recurrence of intraocular inflammation 1 month after injury. d No corneal epithelial defect observed 1 month after the injury on fluorescein staining.

Fig. 3. Changes in CCT and BCVA after injury. BCVA improved 1 week after injury in line with the improvement in corneal edema. CCT, central corneal thickness; BCVA, best-corrected visual acuity.

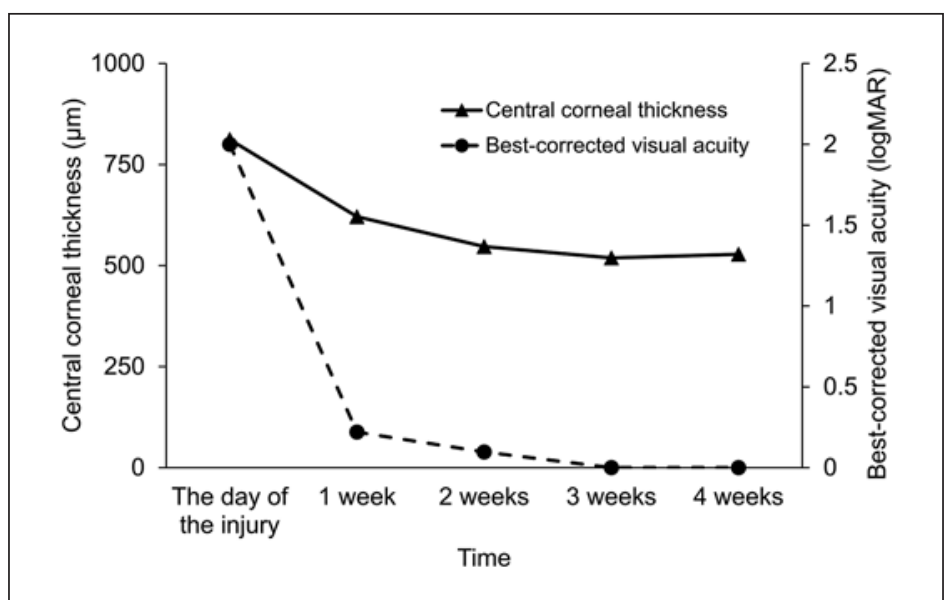

\section{Discussion/Conclusion}

We described a case of corneal endothelial dysfunction after exposure to the sap of E. trigona. The patient presented with very low visual acuity owing to corneal edema and 
anterior uveitis during the initial visit. Because there was no corneal epithelial defect and the symptoms responded rapidly to topical steroid and antibiotic administration, the condition probably involved inflammation to a greater extent rather than infection. Increase in the CCT due to dysfunction of the corneal endothelium decreased gradually after treatment initiation, as shown in Figure 3; this was in contrast to the effect on the ECD, which remained the same. This suggests that exposure to the sap of E. trigona could lead to corneal endothelial cell dysfunction with anterior uveitis immediately after eye injury and that the toxic effect could be transient for up to a month if the condition was treated appropriately and rapidly.

Exposure to the sap of E. trigona could reportedly lead to ocular injuries with a relatively similar clinical course, wherein patients experience conjunctivitis, keratitis, and uveitis without complications in the posterior chamber, soon after exposure [11, 12]. Our patient also showed serious corneal edema with anterior uveitis and no vitreous opacity, 2 days after exposure to the sap of E. trigona. However, in contrast to previous reports by Scott and Karp [11] and Cinar et al. [12], in which patients experienced large epithelial defects or epitheliopathy, no corneal epithelial defect was seen in our case after fluorescein staining despite the presence of anterior uveitis and hypopyon. This suggests that although the toxins in the sap of this genus of plants may cause corneal edema and anterior uveitis, they do not cause corneal epithelial damage. It may be argued that the epithelial defect was probably small and had resolved by the time the patient visited our clinic. However, such an occurrence is highly unlikely since any corneal epithelial damage that leads to severe anterior uveitis and corneal edema can rarely recover in a matter of days, as reported in previous cases $[11,12]$.

The CCT decreased immediately after the initiation of treatment in the current patient and resulted in improvement of visual acuity. Although the prognosis was reportedly good after appropriate treatment with topical antibiotics and steroid instillation $[11,12]$, exposure to the sap of E. trigona could lead to severe dysfunction of the limbal stem cells, mandating corneal transplantation [5]. In the present case, the palisades of Vogt were completely preserved, and the corneal limbus was not damaged. Additionally, the corneal epithelium was intact despite severe anterior uveitis, which also reduced the risk of infectious keratitis. In cases with corneal epithelial defects, bandage contact lenses along with cycloplegics and steroids are useful treatment options for epithelial recovery [11]. Our patient regained visual acuity 1 week after the initiation of treatment and showed complete recovery 1 month after the start of treatment with no complications.

After exposure to the sap of Euphorbia genus, the pH of the ocular surface becomes alkaline [14]. This change in $\mathrm{pH}$ can be helpful in determining the amount of sap that has entered the eye and in predicting the severity of the toxic effect, such as chemical burns [15]. Transient corneal endothelial cell dysfunction may occur following ocular trauma, including chemical burns accompanied with a change in $\mathrm{pH}$ [16]. We did not measure $\mathrm{pH}$ of the ocular surface in our patient and hence could not determine whether the change in $\mathrm{pH}$ could have predicted the course of the disease. Irreversible corneal endothelial cell loss or dysfunction of the limbal stem cells was not observed in our case; this was probably owing to early intervention. Further research on the change in $\mathrm{pH}$ of the ocular surface after injury due to $E$. trigona would be helpful in evaluating the prognosis. Additionally, it would be clinically crucial to wash the ocular surface with a sufficient amount of saline immediately after the injury to normalize the $\mathrm{pH}$.

People who work outside or grow plants of the Euphorbia genus should be careful when handling these plants to prevent the sap from coming into contact with the eye. In case of exposure, close follow-up is required after the injury because of the high risk of concomitant bacterial and fungal keratitis. Ophthalmologists should be aware of the toxicity of the sap of these plants and should start treatment immediately after exposure to prevent irreversible corneal damage.

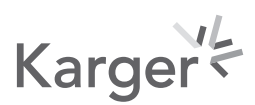


In conclusion, we encountered a patient with severe corneal edema with anterior uveitis that impaired visual acuity after exposure to the sap of E. trigona. It is important to avoid any contact of the sap of E. trigona with the eyes when in vicinity of the plant.

\section{Acknowledgments}

There were no nonauthor contributors requiring acknowledgment.

\section{Statement of Ethics}

This study protocol was reviewed and approved by the Institutional Review Board of Miyata Eye Hospital (Approval No. CS-255). The current study adhered to the tenets of the Declaration of Helsinki. Written informed consent was obtained from the patient for publication.

\section{Conflict of Interest Statement}

The authors have no conflicts of interest to declare.

\section{Funding Sources}

This manuscript did not receive any funding.

\section{Author Contributions}

T.O., Y.M., S.O., and T.I. contributed to the design and conduct of the study. T.O., Y.M., T.M., and R.N. collected, managed, analyzed, and interpreted the data. T.O., Y.M., R.N., T.I., T.M., S.O., and K.M. prepared and reviewed the final manuscript. All authors have read and approved the final version of the manuscript.

\section{Data Availability Statement}

The data that support the findings of this study are available on request from the corresponding author. The data are not publicly available because they contain information that could compromise the privacy of the research participant.

\section{References}

Darlenski R, Kazandjieva J, Tsankov N. Phytodermatitis to Euphorbia trigona. Skinmed. 2014;12(4):253-5.

Elmas ÖF. Irritant contact dermatitis due to Euphorbia trigona. Contact Dermatitis. 2020;82(4):234-5.

3 Merani R, Sa-Ngiampornpanit T, Kerdraon Y, Billson F, McClellan KA. Euphorbia lactea sap keratouveitis: case report and review of the literature. Cornea. 2007;26(6):749-52.

4 Tada M, Seki H. Toxic diterpernes from Euphorbia trigona (saiunkaku: an indoor foliafe plant in Japan). Agric Biol Chem. 1989;53(2):425-30.

5 Biedner BZ, Sachs U, Witztum A. Euphorbia peplus latex keratoconjunctivitis. Ann Ophthalmol. 1981;13(6):739-40. 
6 Eke T, Al-Husainy S, Raynor MK. The spectrum of ocular inflammation caused by Euphorbia plant sap. Arch Ophthalmol. 2000;118(1):13-6.

7 Sofat BK, Sood GC, Chandel RD, Mehrotra SK. Euphorbia royleana latex keratitis. Am J Ophthalmol. 1972;74(4): 634-7.

8 Bessero AC, Achache F, Guex-Crosier Y. Keratouveitis caused by sap of Euphorbia myrsinites plant. Klin Monbl Augenheilkd. 2008;225(5):451-2.

9 McVeigh K. Ubiquitous Euphorbia is anything but euphoria for the eye: a reminder to get any area of contact with the toxic sap under the tap. Eye. 2018;32(2):243-7.

10 Antcliff RJ, Hodgkins PR, Bowman R, Keast-Butler J. Euphorbia lathyris latex keratoconjunctivitis. Eye. 1994; 8 (Pt 6):696-8.

11 ScottIU, Karp CL. Euphorbia sap keratopathy: four cases and a possible pathogenic mechanism. BrJ Ophthalmol. 1996;80(9):823-6.

12 Cinar Y, Turkcu FM, Cingu AK, Yuksel H, Sahin M, Sahin A. Keratouveitis caused by Euphorbia trigona sap. Int Ophthalmol. 2014;34(2):285-7.

13 Dutta J, Mukhopadhyay S, Datta H, Sen S. Boston keratoprosthesis restoring vision in an unusual case of endstage limbal stem cell deficiency following exposure to Euphorbia latex. Int Ophthalmol. 2012;32(1):77-9.

14 Ioannidis AS, Papageorgiou KI, Andreou PS. Exposure to Euphorbia lathyris latex resulting in alkaline chemical injury: a case report. J Med Case Rep. 2009;3:115.

15 Fleischman D, Meyer JJ, Fowler WC. Keratouveitis from Euphorbia cyparissias exposure is a temporal phenomenon. Clin Ophthalmol. 2012;6:851-3.

16 Feizi S. Corneal endothelial cell dysfunction: etiologies and management. Ther Adv Ophthalmol. 2018;10: 2515841418815802. 